\title{
Quantum Nature of Vehicle Motion
}

\author{
Zdzisław Pluta, Tadeusz Hryniewicz* \\ Koszalin University of Technology, Raclawicka 15-17, PL 75-620 Koszalin, Poland \\ *E-mail address: Tadeusz.Hryniewicz@tu.koszalin.pl
}

\begin{abstract}
„, The essence of scientific discovery relies on that one looks at the same that is seen by everyone but notices what no one has seen."

Linus Pauling
\end{abstract}

\begin{abstract}
The paper presents a new adequate look on the character of motion of any material body, such as moving vehicle. It has been proved, that motion of material bodies has a quantum character. Due to this their consecutive energetic states are obtained by a jump. The phenomenon of quantum in the reference to vehicle kinetics has been derived by indicating its place on the background of characteristics of whole mechanics. The length of vehicle way in particular phases of its motion is that source characteristics the derivatives of which form the development of motion description of a material solid/body. It has been indicated that the quantum nature of body motion does not depend on its magnitude. Thus, as has been noticed, there is an analogy between the macro-body motion and electron motion which change its energy also by jump, by getting from one orbit (energetic level) to another.
\end{abstract}

Keywords: Vehicle; Motion; Potential field; Space-time; Kinetics; Time constant; Energetic bridge; Static potential field; Kinematic potential field; Length of potential field

\section{INTRODUCTION}

There is an important reason to consider the titled problem. It results from the need for adequate description of the real issue the core of which is the vehicle motion. The paper covers the whole its motion, just from the beginning, through its different stages/phases, until the end or coming to a standstill of the so complex phenomenon. It is worth considering this issue as it has not been described properly, without a notice of a quantum nature of vehicle. Thus many essential elements has been omitted which are important on the ground of recognition of the reality [1].

One should notice that it is impossible to adequately describe motion of the whole vehicle if the theory for this description is taken from the classical mechanics only. It grew on the basis of foundation of continuity of energy changes, called simply the energy [2]. We cannot assume this. The consequence of this paradigm seems to be also polemic. One cannot make use also of this cognitive material nor use it in a rational way. 
The classical interpretation of particular types of motion realized by the vehicle from start to stop cannot be fully acceptable. Consecutive accelerated motions are usually linearized, referred to the courses both of velocity and acceleration. The retarded motion, taking place during vehicle braking, is often treated by analogy. In that case it is non-linear [3], that results from differential equation, of which basic element is a fictional inertia force, according to the d'Alembert rule [4].

The fiction here has got into the structure of the motion description. The uniform motion always excludes the acceleration in these conditions as being zero. That means the body is without inertia as the measure of this magnitude (inertia force) is zero. In the reality the body possesses inertia in any conditions [5].

In classical approach to the motion phenomenon there are no other parameters. Their list is limited to acceleration, the third consecutive physical magnitude, describing this phenomenon. Any attempt to get out from the source characteristics, being the dependence of the path/way length on time, the consecutive fourth magnitude is leading to fiasco because this magnitude assumes the value equal zero.

It results from the description of these example artifacts of classical mechanics that it is necessary to undertake an action to change this state. Such operations have been undertaken earlier and their results have been presented elsewhere [6-11]. In this work we are going to include that approach into the structure of adequate description of vehicle motion, thus regarding quantum nature of the phenomenon.

The description will be focused on kinetics [12,13]. Other characteristics of the considered object will not be presented, what results evidently from the limited frames of the paper. Anyway, to clarify that question, the classification of all fields/branches falling in general mechanics will be performed. By the way, also the kinetics will be clearly defined.

\section{KINETICS OF VEHICLE ON THE BACKGROUND OF WHOLE MECHANICS CHARACTERISTICS}

The presented classification of mechanics (Fig. 1) possesses quite a new structure resulting from looking at the reality from the viewpoint of quantum behaviour of a material body, where real behaviours occur. Actuated by that supreme rule, the whole mechanics may be divided into three basic fields: energetics, dynamics, and kinetics.

Energetics should consider energetic analysis of states of a material body, with the states occurring when the body is at rest, as well as during the uniform body motion [2]. Therefore the statics and kinematics may be separated. Then the statics should be divided into stabilnics and astabilnics. It results from the fact that the static state of a body may be stable or unstable.

Dynamics of a solid is directing its analysis on the behaviour of a body in variable motion, that is between the neighbouring energetic states, i.e. in the space-time. One should admit that both energetics and dynamics form such field of mechanics, which in the description refers not to a material point but to a real body possessing a mass.

There is the third field of mechanics, the kinetics. And here, within its limits, the behaviour of a model body or a material point is considered. Due to this reason/assumption, all considered magnitudes are massless, i.e. without a mass in its analytical structure. Therefore, for example, the following magnitudes like a path length, velocity, and acceleration are taken into account. All they characterize both the variable motion, and uniform motion providing the following types of kinetics: dynamic, and kinematic one. 
Kinetics is just the main issue of the considerations, devoted to the quantum nature of a vehicle motion.

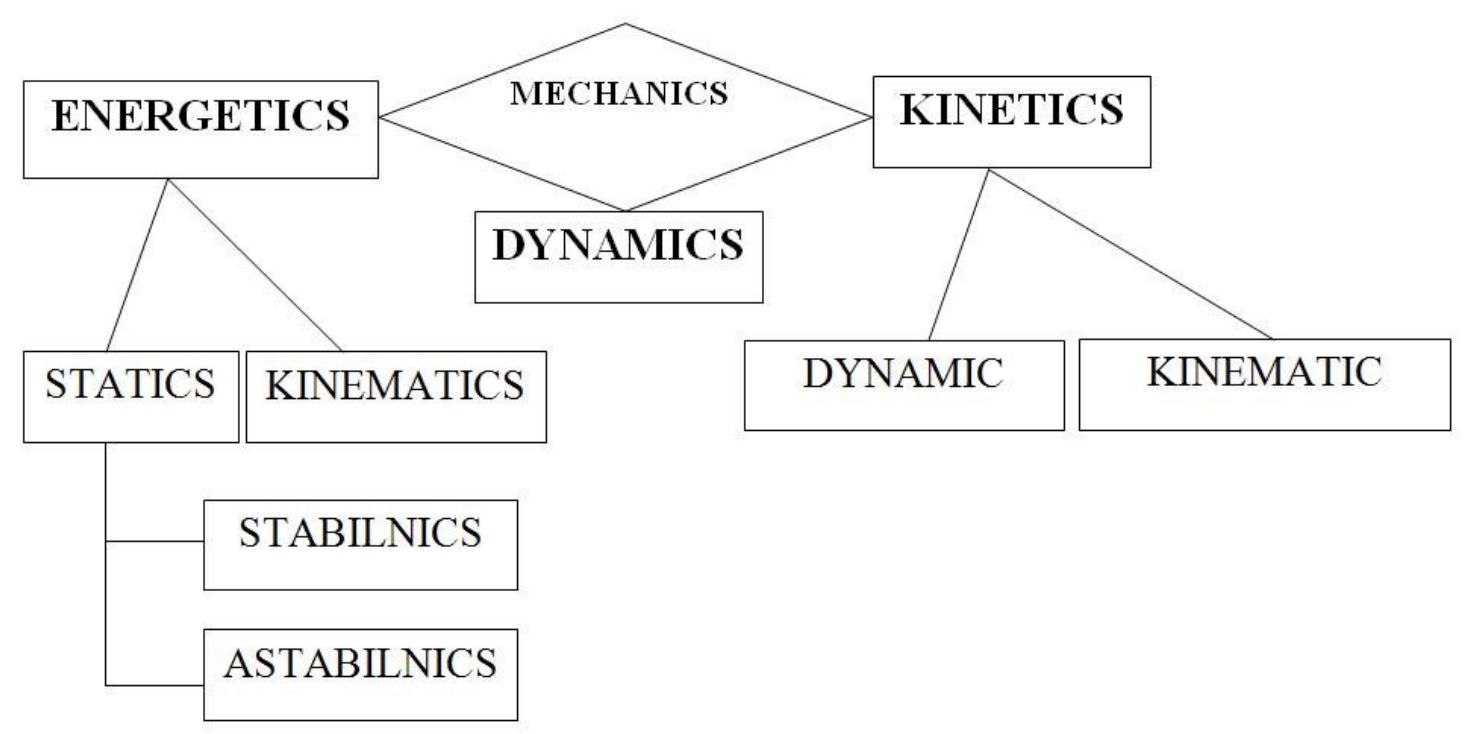

Fig. 1. Classification of mechanics.

\section{PATH LENGTH OF VEHICLE IN PATICULAR PHASES OF ITS MOTION}

The most simple course of vehicle motion covers just three phases, consisting of: start up (accelerated variable motion), uniform motion, and braking, during which a retarded variable motion takes place. In fact the vehicle passes through several two-stage cycles before it moves with a determined optimum and constant velocity, that is a developed uniform motion, after which finally the braking occurs, and returning of the vehicle to the primary state. These two-step behaviours cover the following two, one after another, motions: accelerated, and then - uniform motion.

This pictorial, quite general description of the vehicle motion is just an introduction to precision, in-depth description of the phenomenon in the whole cognitive area. The path/way length is the first, the most important, source characteristics of the considered phenomenon. Next will be derived the characteristics, and the quantity interval cannot be closed as well. The acceleration will be the third consecutive magnitude, but not the last one.

The primary equation of the path length has the following differential form:

$$
d s= \pm \frac{\partial s}{\partial t} d t
$$

where $d s$ - means total differential of path, $\partial s$ - is partial differential of this magnitude, $d t-$ total differential of time, and $\partial t$ - its partial differential.

The signs $( \pm)$ are algebraic operators, which possess their separate functions. The sign $(+)$ has only a formal meaning because it confirms the physical sense of the phenomenon description. The sign (-) is to derive a physical sense of determined record of the considered 
phenomenon [12-14]. That occurs at the interaction of retarded motion. The accelerated motion does not require such a formal procedure.

That solution of equation (1) is given in [15]. For an accelerated motion the solution has the following form:

$$
S=S_{1}\left(e^{\frac{t}{T_{1}}}-1\right)
$$

and the retarded motion is characterized by the following dependence of the path/way length on time:

$$
s=2 s_{2}\left(1-e^{-\frac{t}{T_{2}}}\right)
$$

Just between these types of variable motion the uniform motion occurs where the path length varies according to the relationship:

$$
s=v_{1} \cdot t
$$

in the interval $\left\langle 0, s_{1}^{*}\right\rangle$, where $s_{1}^{*}$ is a kinematic length of the potential field.

The courses of path length $s$ of vehicle in the whole its shortest cycle (Fig. 2) are - as results from the higher records - essentially differentiated. In the accelerated motion that course is exponentially progressive in character. The second phase of motion is illustrated by a straight line, of which the inclination against time axis corresponds with the velocity $v_{l}$.

The third and last phase of the motion refers to a degressive exponential course of the considered magnitude.

Symbol $s_{1}$ means a total path length travelled by the object in accelerated motion. Notation $T_{1}$ corresponds with a time constant for this motion. That constant means the transition time of a body to a neighbouring potential field with an identical initial velocity $v_{o}$. Thus the presented magnitudes are connected one with each other in the following manner:

$$
v_{o}=\frac{s_{1}}{T_{1}}
$$

One should explain now the introduced notion of the potential field; that means the place where the body possesses a determined energetic state. At the beginning, in the state of immobility, it was at the static potential field (SPF). Here it possesses a static energetic state. Further on it entered into the space-time (dotted area in Fig. 2), where the phenomenon of accelerated variable motion occurred. After the transition over this combination of time and space, or just the space-time, the body enters onto the neighbouring kinematic potential field (KPF). 


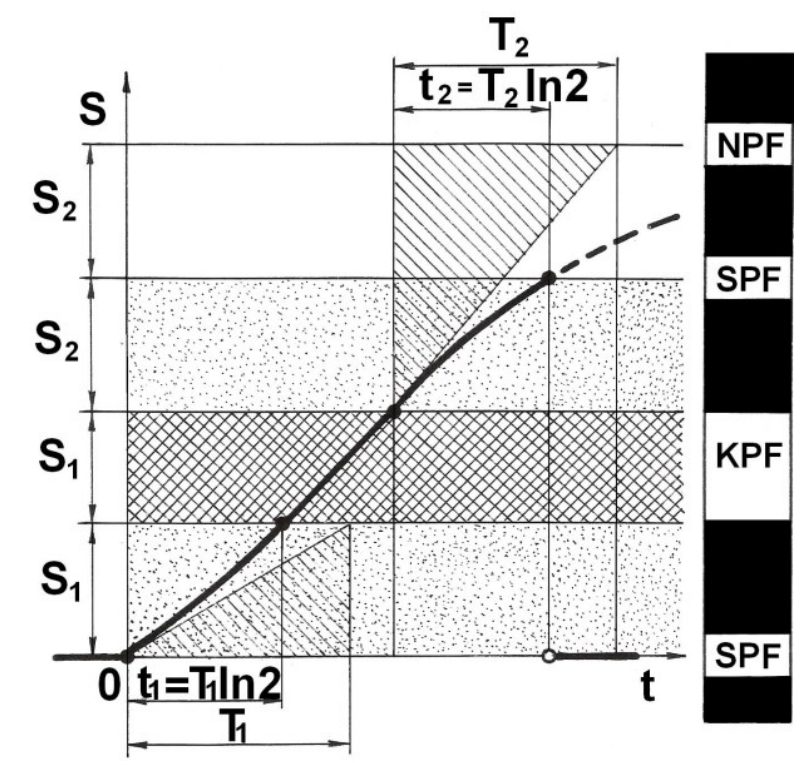

Fig. 2. Course of the path length $s$ of vehicle in the whole shortest its cycle.

Symbol $s_{1}$ means a total path length travelled by the object in accelerated motion. Notation $T_{1}$ corresponds with a time constant for this motion. That constant means the transition time of a body to a neighbouring potential field with an identical initial velocity $v_{o}$. Thus the presented magnitudes are connected one with each other in the following manner:

$$
v_{o}=\frac{s_{1}}{T_{1}}
$$

One should explain now the introduced notion of the potential field; that means the place where the body possesses a determined energetic state. At the beginning, in the state of immobility, it was at the static potential field (SPF). Here it possesses a static energetic state. Further on it entered into the space-time (dotted area in Fig. 2), where the phenomenon of accelerated variable motion occurred. After the transition over this combination of time and space, or just the space-time, the body enters onto the neighbouring kinematic potential field (KPF). From this very moment the uniform motion proceeds with the vehicle possessing a kinematic energetic state. The kinematic potential field corresponds with the oblique squared area in Fig. 2. Here the path length varies in accordance with the dependence (4) that is straight proportionally to the velocity $v_{l}$. That was velocity, corresponding with the body velocity at the end of the first space-time, the body moved in this potential field.

After the transition through this field, the considered body entered the next space-time (the dotted field in Fig. 2). At the beginning it possessed the velocity $v_{1}$, which was connected with the length of the space-time $s_{2}$ and the time constant $T_{2}$ by the following relationship:

$$
v_{1}=\frac{2 s_{2}}{T_{2}}
$$

It is worth noting that the time constant $T_{2}$ is now at the moment/hight of nominal potential field (NPF), that is an asymptote, to which the curve corresponding with the course 
of path length approaches. As can be seen, from the asymptote side there is an improper space-time, where the course of considered magnitude has been marked only formally (dashed line). It has no physical sense. A real course of the path length terminates at the border between the last oblique squared proper/virtual space-time (dotted area) and that improper space-time.

In this motion cycle, the shortest cycle, the symmetry of terminal space-times occurs. Therefore the identity/equality of time constants occurs, i.e. $T_{1}=T_{2}$. One may also determine the transition time through these space-times. It will be also identical, and it may be determined by substituting $s_{1}$ to the formula (2), and $s_{2}$ to the formula (3) with these magnitudes being also equal/identical $\left(s_{1}=s_{2}\right)$. Thus

$$
t_{1}=t_{2}=T_{1} \ln 2=T_{2} \ln 2
$$

Just at this stage of considerations the quantum (jump) nature of the vehicle motion phenomenon may be perceived/noticed. The limits of space-time, where the phenomenon of variable motion (referred to the properly understood dynamics) takes place, are the determined potential fields. These fields, in turn, clearly determine the defined energetic states of the vehicle which, as can be noticed, vary by a quantum.

Therefore the energy varies by quantum independent on the body size. Unfortunately the classical mechanics used to inform that the energy of macroscopic bodies varies in a continuous way. These statements are also presented in the latest literature in this scientific branch [16].

\section{COURSES OF DERIVATIVES OF KINETIC MAGNITUDES}

There are several derivatives of kinetic magnitudes. Their number is not limited to two only, which are the velocity and acceleration, as it takes place in the classical theory. This kind of set is on one-side closed, from the side of source with the source magnitude being the path length $s$. Then it has an open character. Its further elements, being derivative kinetic magnitudes, have no limits.

Here the courses of only three example magnitudes of this type have been analyzed. The velocity $v$, acceleration $a$, and impulse $i$ have been taken into account. A new physical magnitude has appeared which even occurs in the classical mechanics, but has no its adequate meaning. It is identified with the impulse of force though as to the essence it refers simply to the impetus.

They were the artificial compositions the introduction of which resulted from the impossibility to form further derivative physical magnitudes, with the acceleration being the last one the second derivative magnitude of the classical mechanics.

The mentioned three derivative kinetic magnitudes (velocity $v$, acceleration $a$, and impulse $i$ ), being consecutively the first one, the second, and third derivatives of the path/way length have the following their functional forms for the accelerated motion:

$$
v=\frac{d s}{d t}=\frac{s_{1}}{T_{1}} e^{\frac{t}{T_{1}}}=v_{o} e^{\frac{t}{T_{1}}}
$$




$$
\begin{gathered}
a=\frac{d^{2} s}{d t^{2}}=\frac{d v}{d t}=\frac{s_{1}}{T_{1}^{2}} e^{\frac{t}{T_{1}}}=\frac{v_{o}}{T_{1}} e^{\frac{t}{T_{1}}}=a_{o} e^{\frac{t}{T_{1}}} \\
i=\frac{d^{3} s}{d t^{3}}=\frac{d^{2} v}{d t^{2}}=\frac{d a}{d t}=\frac{s_{1}}{T_{1}^{3}} e^{\frac{t}{T_{1}}}=\frac{v_{o}}{T_{1}^{2}} e^{\frac{t}{T_{1}}}=\frac{a_{o}}{T_{1}} e^{\frac{t}{T_{1}}}=i_{o} e^{\frac{t}{T_{1}}}
\end{gathered}
$$

For a retarded motion these all the kinetic magnitudes have the following functional records:

$$
\begin{gathered}
v=\frac{d s}{d t}=\frac{2 s_{2}}{T_{2}} e^{-\frac{t}{T_{2}}}=v_{1} e^{-\frac{t}{T_{2}}} \\
a=-\frac{d^{2} s}{d t^{2}}=-\frac{d v}{d t}=\frac{2 s_{2}}{T_{2}^{2}} e^{-\frac{t}{T_{2}}}=\frac{v_{1}}{T_{2}} e^{-\frac{t}{T_{2}}}=a_{1} e^{-\frac{t}{T_{2}}} \\
i=-\frac{d^{3} s}{d t^{3}}=-\frac{d^{2} v}{d t^{2}}=-\frac{d a}{d t}=\frac{2 s_{2}}{T_{2}^{3}} e^{-\frac{t}{T_{2}}}=\frac{v_{1}}{T_{2}^{2}} e^{-\frac{t}{T_{2}}}=\frac{a_{1}}{T_{2}} e^{-\frac{t}{T_{2}}}=i_{1} e^{-\frac{t}{T_{2}}}
\end{gathered}
$$

For the uniform motion, occurring between these two terminal phenomena, i.e. the accelerated motion and retarded motion, all the separated derivative magnitudes will be constant and invariable ( $v=v_{1}=$ const., $a=a_{1}=$ const., $i=i_{1}=$ const.). They result from substituting the time $t_{l}=\mathrm{T}_{l} \ln 2$ to (8), (9), (10), and the results of these operations are presented as follows: $v_{l}=2 v_{o}, a_{l}=2 a_{o}, i_{1}=2 i_{o}$.

All these courses have been illustrated graphically in Fig. 3. Here the energetic links of the terminal variables of courses of derivative magnitudes are clearly visible. They are alike energetic bridges, connecting terminals of the considered cycle of vehicle motion.

As may be noticed, between these terminals, there are no zero values of acceleration nor impulse. There is a continuity, being a natural continuity of the courses. Only at the moments of entrance to the space-time, as well as the exit/departure of it, the characteristic jumps of these magnitudes have appeared.

The presented descriptions again do not fit to the theory of classical mechanics. However, as can be noticed, they possess the adequate character, referred to the real course of this motion cycle of considered object. It is worth admitting that this theory restores the existence of inertia force in the uniform motion, that results from this, that the acceleration is determined here, being constant and positive. Therefore in the kinematic balance that force should be taken into account that is not done according to the theory of classical mechanics. It is time now to change the description of the body motion to be adequate and conformable with the natural reality. 


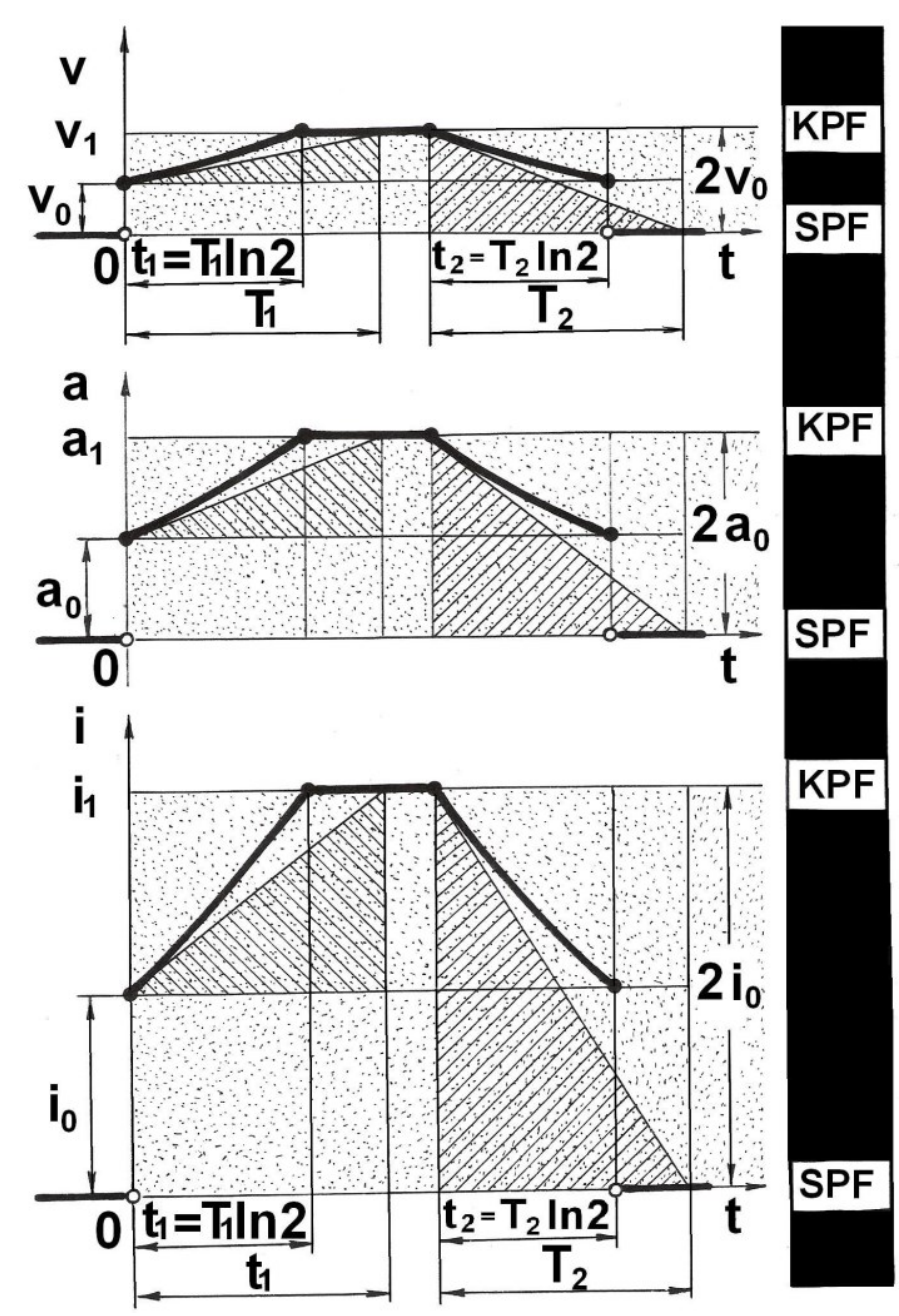

Fig. 3. Courses of three derivatives of kinetic magnitudes: velocity $v$, acceleration $a$, and impulse $i$.

\section{SOURCE AND THE FIRST DERIVATIVE CHARACTERISTICS OF VEHICLE MOTION FOR A DEVELOPED CYCLE}

The source characteristics of vehicle motion for a developed cycle is just this dependence of path length $s$ on time $t$ (Fig. 4). This cycle may possess several stages of motions: accelerated and uniform, but final, one stage, covering the retarded motion, closes that whole cycle of motion. The picture (Fig. 4) presents two such two-type stages and a final stage. There are no terminal symmetries of the space-time. Each space-time is different.

Dependence of the path length on time for the first space-time is analogous according to the formula (2). For the second space-time that dependence has the following form:

$$
S=S_{2}\left(e^{\frac{t}{T_{2}}}-1\right)
$$

whereas the third space-time is characterized by the dependence of type: 


$$
s=2 s_{2}\left(1-e^{-\frac{t}{T_{3}}}\right)
$$

It is worth noticing the quantum nature of the phenomenon of vehicle motion. There are energetic breaks clearly visible (dotted areas in Fig. 4), where the analyzed complex material body does not possess the energy, moving with an accelerated motion, and at the very end, with a retarded motion. These breaks have been separated by kinematic potential fields (oblique squared areas in Fig. 4), and terminal points of the whole motion correspond with the static potential fields.

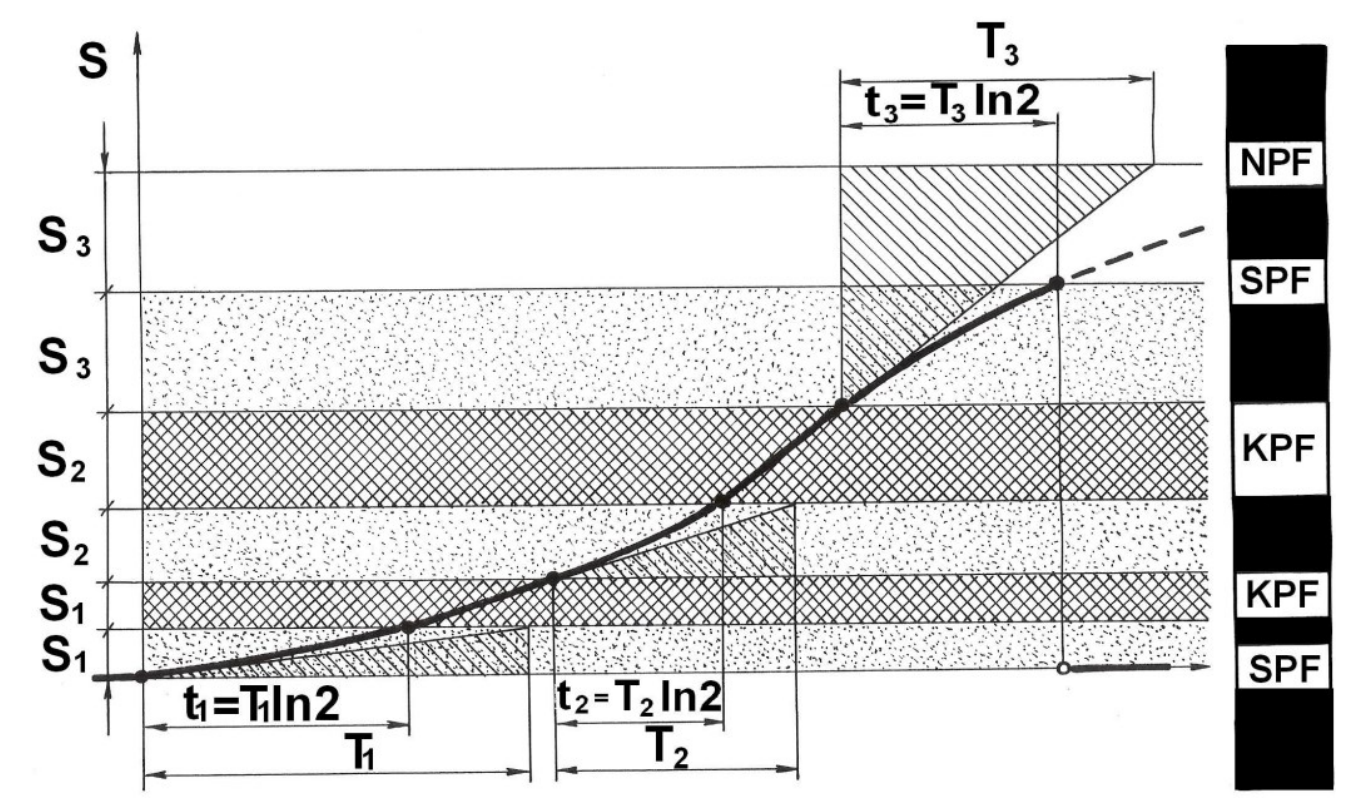

Fig. 4. Dependence of the path length $s$ on time $t$ for developed cycle of vehicle motion.

One may notice also an analogy occurring between the motion of this macro-body and the motion of electrons which vary their energy by jump, getting with this to other orbits (energetic levels). Thus different possible electron states are the energetic breaks of some kind. They are just these space-times to be penetrated by these elementary particles.

The second time characteristics of the developed cycle of vehicle motion comes to the dependence of the velocity $v$ on time $t$ (Fig. 5). For the first space-time this dependence has the form of formula (8). Further on, at the first kinematic potential field, possessing the length $s_{1}^{*}$, the velocity $v=v_{1}=$ const. Through the second space-time, the analyzed body penetrates with the velocity, of which the dependence on time is the result of differentiating the formula (14) and takes the following form:

$$
v=\frac{d s}{d t}=\frac{s_{2}}{T_{2}} e^{\frac{t}{T_{2}}}=v_{1} e^{\frac{t}{T_{2}}}
$$

On the next, second kinematic potential field, of the length $s_{2}^{*}, v=v_{2}=$ const. 


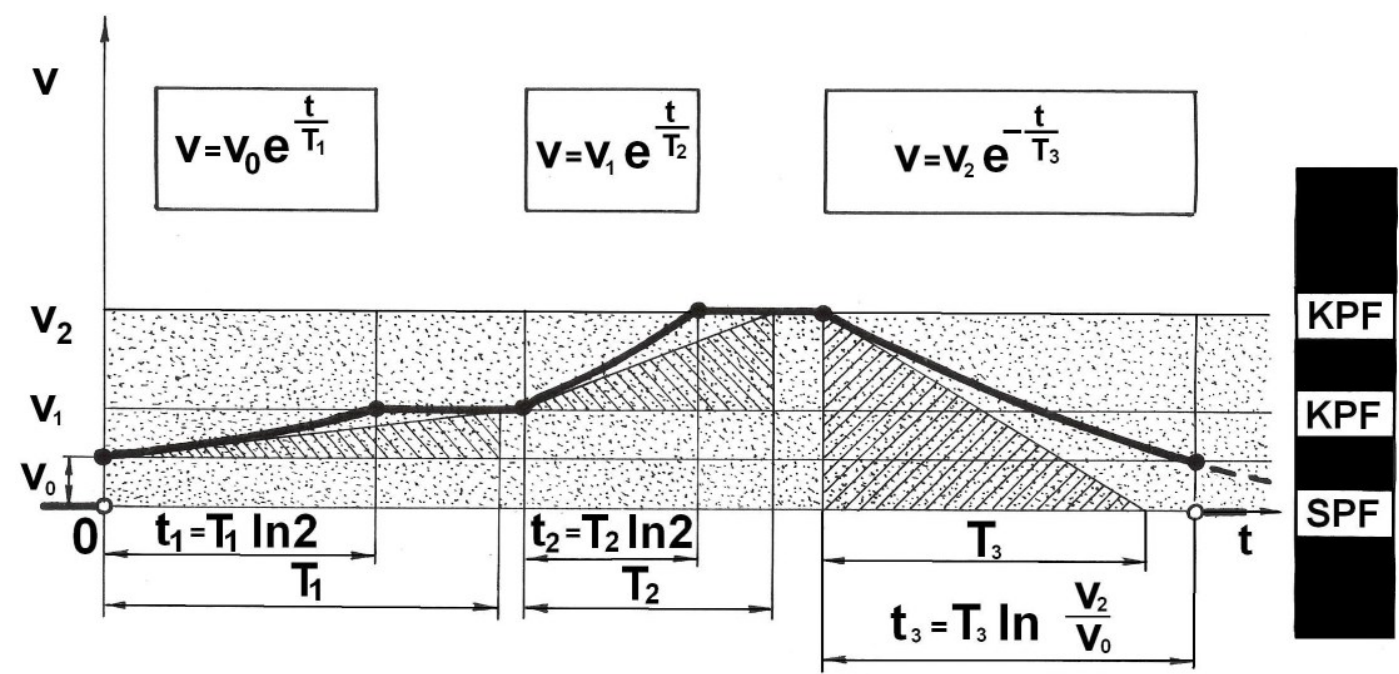

Fig. 5. Dependence of velocity $v$ on time $t$ for a developed cycle of vehicle motion.

Now the considered body returns to the initial state, where $v=v_{o}$. It passes with a retarded motion, described by the relationship which, in turn, results from differentiation of formula (15). Here is the result of this mathematical operation:

$$
v=\frac{d s}{d t}=\frac{2 s_{3}}{T_{3}} e^{-\frac{t}{T_{3}}}=v_{2} e^{-\frac{t}{T_{3}}}
$$

Time constant $T_{3}$ results from the connection of the path length $s_{3}$ with the velocity $v_{2}$, that is

$$
v_{2}=\frac{2 s_{3}}{T_{3}}
$$

Now time $t_{3}$ of the retarded motion of vehicle is to be determined. Thus first the constant $T_{3}$ should be connected with a final velocity, equal to the initial velocity $v_{o}$. It is obtained straight from the equation (17), by substituting $t=t_{3}, v=v_{o}$, to it, that is

$$
v_{o}=v_{2} e^{-\frac{t_{3}}{T_{3}}}
$$

and after proper transformations:

$$
T_{3}=\frac{t_{3}}{\ln \frac{v_{2}}{v_{o}}}
$$

The time of the final retarded vehicle motion results from this, then:

$$
t_{3}=T_{3} \ln \frac{v_{2}}{v_{o}}
$$


The courses of vehicle velocities are succeeding confirmation of the fact that its motion has quantum (jump) character. The energetic levels here are clearly marked with the velocity being constant and invariable. These levels are separated by space-times (energetic breaks), where the dynamics of the considered body takes place. That means here is no equilibrium. One may admit: everything varies in the space-times.

\section{CONCLUSION}

Summing up it is worth underlining the essence of scientific discovery which was very accurately formulated by Pauling. It is not enough to look and see. One should notice the sense and essence of the matter.

It results from the analysis carried out that the vehicle motion possesses a quantum nature. This refers to each material body which with free or non-free (forced) motion passes from the rest state through the space-times, finally returning to the primary state. Thus not a size/magnitude of a material body but its real natural behaviour decides of this.

Limited frames of the work do not allow to develop the issue. Therefore at present the explanation of the essence of quantitatives of the vehicle motion is given. That was performed on the basis of kinetic characteristics. The described problem is to be developed later in the following publications.

\section{References}

[1] Zdzisław Pluta, Tadeusz Hryniewicz, International Letters of Chemistry, Physics and Astronomy 7(1) (2013) 45-59.

[2] Zdzisław Pluta, Tadeusz Hryniewicz, Journal of Modern Physics 3(7) (2012) 625-635; DOI: $10.4236 /$ jmp.2012.37086.

[3] J. Lanzendoerfer, Technika Motoryzacyjna 2 (1974) 21-25.

[4] Zdzisław Pluta, Tadeusz Hryniewicz, International Letters of Chemistry, Physics and Astronomy 7(2) (2013) 85-101.

[5] Zdzisław Pluta, Tadeusz Hryniewicz, International Letters of Chemistry, Physics and Astronomy 4 (2013) 56-72.

[6] Zdzisław Pluta, LAB (Laboratoria, Aparatura, Badania) 2 (2004) 50-52.

[7] Zdzisław Pluta, LAB (Laboratoria, Aparatura, Badania) 6 (2004) 42-46.

[8] Zdzisław Pluta, LAB (Laboratoria, Aparatura, Badania) 2 (2005) 45-48.

[9] Zdzisław Pluta, LAB (Laboratoria, Aparatura, Badania) 4 (2005) 43-46.

[10] Zdzisław Pluta, LAB (Laboratoria, Aparatura, Badania) 6 (2005) 30-34.

[11] Zdzisław Pluta, Energetyka 7 (2005) 496-501.

[12] Zdzisław Pluta, Tadeusz Hryniewicz, International Letters of Chemistry, Physics and Astronomy 3 (2013) 85-100.

[13] Zdzisław Pluta, Tadeusz Hryniewicz, Intern. J. Adv. Mater. Technol. 62(5) (2012) 529-542, DOI: 10.1007/s00170-011-3813-5. 
[14] Zdzisław Pluta, Tadeusz Hryniewicz, International Letters of Chemistry, Physics and Astronomy 5 (2012) 35-45.

[15] Zdzisław Pluta, Tadeusz Hryniewicz, International Letters of Chemistry, Physics and Astronomy 6 (2013) 116-136.

[16] J. R. Taylor, Classical mechanics (in Plish), Vols. 1 and 2. (transl. From English), 1st ed. Wydawnictwo Naukowe PWN SA, Warszawa 2006. 